Courrier du Centre International Blaise-

Pascal

38-39 | 2017-2018

Varia

\title{
Pascal, Port-Royal et le P. Pirot
}

Olivier Jouslin

\section{(2) OpenEdition}

Journals

Édition électronique

URL : https://journals.openedition.org/ccibp/1295

DOI : $10.4000 /$ ccibp. 1295

ISSN : 2493-7460

\section{Éditeur}

Centre international Blaise Pascal

\section{Édition imprimée}

Date de publication : 1 janvier 2019

Pagination : 23-45

ISBN : 978-2-84516-897-8

ISSN : 0249-6674

Référence électronique

Olivier Jouslin, «Pascal, Port-Royal et le P. Pirot », Courrier du Centre International Blaise-Pascal [En ligne], 38-39 | 2017-2018, mis en ligne le 17 juin 2021, consulté le 03 mai 2022. URL : http:// journals.openedition.org/ccibp/1295; DOI : https://doi.org/10.4000/ccibp.1295

Ce document a été généré automatiquement le 3 mai 2022.

Centre international Blaise Pascal 


\title{
Pascal, Port-Royal et le P. Pirot
}

\author{
Olivier Jouslin
}

1 Un large pan des Provinciales s'attaque ouvertement à la morale relâchée attribuée aux jésuites. Certaines maximes produites par Pascal dans ses lettres émeuvent le public pendant que, dans les milieux ecclésiastiques, on cherche à obtenir leur condamnation...

Ce fut alors que les défenseurs de ces nouvelles doctrines, les voyant condamnées par les prélats, et décriées parmi les peuples, se persuadèrent que pour relever le crédit de leurs casuistes, il fallait les soutenir par quelque ouvrage considérable ${ }^{1}$.

2 Les auteurs du Septième Écrit des curés de Paris expliquent ainsi la mise en circulation de l'Apologie pour les casuistes ${ }^{2}$ dont l'influence sera en effet considérable dans l'histoire de la lutte des rigoristes contre la nouvelle casuistique. L'ouvrage circule à la midécembre 1657, il est anonyme mais, très tôt, on apprend qu'il est de Georges Pirot (1599-1669) ${ }^{3}$, régent de théologie au collège de Clermont.

3 L'Apologie entre dans la catégorie des réponses générales aux Provinciales. Elle défend les nouveaux casuistes en optant pour un format relativement étendu (191 pages in $4^{\circ}$ ). Pirot y propose, en cinquante-quatre «Objections» numérotées et suivies de leur "Réponse ", une réfutation des principales accusations véhiculées par les lettres pascaliennes contre la perversion morale attribuée à certains jésuites. L'ouvrage se clôt sur un «Avis des jansénistes à ceux qui répondent à leurs lettres ». Pirot annonce son dessein et sa méthode dès le début de son texte :

Pour le faire [réfuter les Provinciales et les jansénistes] avec quelque méthode, je réfuterai premièrement les calomnies dont ils noircissent la profession de casuiste, je répondrai ensuite à ce qu'ils opposent contre les principes généraux de la morale. Enfin, je suivrai à peu près l'ordre de leurs lettres depuis la quatrième jusques à la quinzième, où ils finissent leurs injures contre cette sorte de théologie ${ }^{4}$.

4 Les analyses de la campagne des Provinciales, qu'elles soient ou non favorables aux jésuites, évacuent souvent très rapidement cette pièce. Cette mise au ban peut s'expliquer par sa maladresse de ton mais aussi par l'option d'une logique polémique qu'on peut considérer comme jusqu'au-boutiste. Pirot assume et défend en effet les positions les plus «ouvertes», ou «laxistes», comme on voudra l'entendre, des casuistes modernes. Pourtant l'histoire des relations de ce texte avec Port-Royal et avec 
Pascal mérite qu'on s'y arrête et - peut-être - qu'on révise le peu d'attention qu'on a l'habitude de porter à l'ouvrage de Pirot.

5 L'histoire familiale des Pascal nous apprend d'ailleurs que des relations d'ordre presque privées lièrent les Pascal et l'Apologie. En effet, le Père Amable de Frétat, le cousin jésuite des Périer qui avait essayé de prévenir Pascal des dangers qu'il encourait au moment de la mise en circulation des Provinciales, sera exilé à Moulins avec défense de confesser pour s'être opposé à la publication de l'Apologie $e^{5}$. Mais, au-delà d'un rapport strictement événementiel entre les Pascal et cette défense, cette pièce se révèle fascinante dans l'optique d'une étude des rapports directs qu'entretiennent Port-Royal et ses contradicteurs car les hasards de la conservation ont fait que la Bibliothèque Mazarine possède un exemplaire de l'Apologie comportant des annotations anciennes. Or, la tradition rapporte que certaines des annotations émanent de la main de Pascal lui-même ${ }^{6}$.

6 L'exemplaire 4551, conservé dans la Grande Réserve de la bibliothèque parisienne est issu du fonds légué par Prosper Faugère. Il provient de pièces issues de la bibliothèque de Pascal, léguées directement par la famille Périer à l'abbaye de Saint-Jean-d'Angély. Un des tirages originaux de l'Apologie y a été serré, en compagnie de papiers anciens, dans un dossier contenu dans un portefeuille de velours rouge. Des annotations manuscrites accompagnent et jugent le texte de Pirot:

On conviendra que cette injure mal déguisée sous sa grossière et cynique

expression, est absolument dépourvue d'esprit et de goût ${ }^{7}$.

7 Tout en déniant tout intérêt à la pièce, l'auteur de ces mots (Faugère lui-même ?) joint un papier où une ligne manuscrite ancienne - authentifiée par une ligne au crayon comme émanant de la main même de Nicole - indique que des notes sont de Pascal.

8 L'intérêt de cette pièce tient donc au fait qu'elle offre pour l'étude de la polémique liée aux Provinciales un objet d'étude exceptionnel : un tirage de l'Apologie pour les casuistes porte les marques de la façon dont on lit à Port-Royal et dans l'entourage proche de Pascal les textes ennemis. Mieux encore, Pascal lui-même annote peut-être le texte, ou le fait annoter, ce qui est bien la marque de l'intérêt et de l'attention qu'il porte aux textes de l'autre camp 8 .

9 Il ne s'agira pas ici de mener au sens strict du terme une étude paléographique canonique pour décider de qui émanent les lignes manuscrites qui émaillent le texte du jésuite. On cherchera plutôt à scruter les annotations pour cerner - à travers l'étude d'une pratique de lecture - à la fois une attitude polémique et le mode d'expression d'une opposition profonde entre deux adversaires.

10 Ce projet passe par une étude précise des annotations comme marques matérielles. On essayera d'abord d'en présenter une typologie pensée sur un mode dynamique : il faut décrire les annotations pour aborder la question des limites de cette démarche dans le cas singulier de l'exemplaire qui nous occupe. On s'attachera ensuite à préciser les sens que peut prendre la lecture dont ces annotations portent la marque.

\section{Une typologie et ses limites}

11 L'exemplaire contient environ 166 annotations. 86,9\% des 191 pages de l'Apologie comportent donc au moins une marque de lecture. Elles sont réparties sur la totalité du texte, aucune partie de celui-ci n'est oubliée par les lecteurs. Aucun espace précis dans 
le texte ne semble d'emblée concentrer un nombre plus important d'annotations que les autres. L'ouvrage a donc été lu cursivement au moins une fois et annoté au cours de la lecture. Certaines écritures, nettes au début, portent la marque du relâchement consécutif à la fatigue de la main qui tient la plume. On note encore des différences de couleur d'encre et des écritures distinctes. Se dessine déjà ici l'image de l'officine des lecteurs de Port-Royal en contexte polémique: plusieurs plumes sont à l'œuvre, l'opération de lecture s'est étendue sur une période dont l'étendue n'est pas précisément mesurable mais qui exclut une lecture singulière ${ }^{9}$. On suppose que furent à l'œuvre des lecteurs de premier rang et des lecteurs secondaires. L'annotateur principal n'a pas été prévenu qu'une partie précise de l'ouvrage devait concentrer son attention puisque ses annotations sont disséminées sur tout le texte. Il faut maintenant passer à la description précise de celles-ci.

Elles résident, pour une part, en de classiques marques de lecture : on trouve des traits marginaux, des passages du texte sont soulignés, d'autres sont annotés. On remarque encore deux autres niveaux d'annotations qui ne sont pas précisément des marques de lecture. Elles ne semblent pas émaner du lecteur principal et portent la marque qu'un autre travail a été effectué sur le texte dont le classement typologique qui suit rendra compte.

13 La typologie proposée ici est un classement qui repose sur un préalable, presque un scénario. On part du point de vue suivant: les lecteurs de Port-Royal annotent les endroits du texte qu'ils remarquent. Et ils remarquent le pire. On suppose donc qu'ils sont choqués par ce qu'ils lisent et que leurs annotations portent la marque du scandale. Toute annotation est donc à lire comme un commentaire négatif, une réaction, et parfois un choc.

\section{a. Proposition de typologie, un scénario du rapport de Port-Royal aux textes adverses}

14 La typologie proposée repose sur un effet d'accentuation : on ira d'un lecteur qu'on suppose interpellé à un lecteur proprement scandalisé.

\section{Le premier niveau d'annotation : les annotations non verbales}

15 Le premier niveau d'annotation comporte des annotations non verbales associées ou non à des mots. On peut les distinguer en cinq catégories.

\section{Les traits latéraux}

16 Ils consistent en des traits marginaux qui attirent l'attention verticalement, de façon externe au texte, sur une partie de la page. Les traits marginaux simples sont typiques de la lecture précise et cursive. Ces traits simples supposent que c'est cet endroit du texte, à un moment de la lecture, qui attire l'attention et suscite une réaction. Celui qui voit ces marques se trouve ici confronté à une première limite dans la volonté de les comprendre : le trait latéral comporte une part d'ambiguïté dans la mesure où, à la différence du crochet par exemple, il ne permet pas toujours de décider exactement de la partie du texte qui fait réagir l'annotateur. Dans certains cas l'ambiguïté peut conduire à se demander si une « objection » de Pirot doit être prise en compte dans son 
entier ${ }^{10}$. L'annotateur peut d'ailleurs éprouver le besoin de préciser quelle partie exacte du texte est visée par le trait en la délimitant par un crochet interne ${ }^{11}$.

Un long trait est parfois redoublé par un autre plus court, qui isole une partie plus précise du texte déjà marqué. Un trait redoublé viendra ainsi renforcer un trait plus long pour marquer un passage où une abbaye de vierges chastes (Pirot vise les religieuses de Port-Royal) se change en un sérail de filles impures ${ }^{12}$. La réaction scandalisée franchit une nouvelle étape lorsque l'ensemble d'un passage est marqué intégralement - d'un double trait. Le cas des certaines pages est intéressant dans la mesure où elles associent des passages marqués par un double trait à d'autres, marqués par un simple trait ${ }^{13}$. Ce cas permet d'établir sur la même page une échelle d'intensité dans la vigueur de la réaction. Le scandale est à son comble dans le cas d'un hapax où un passage du texte est marqué triplement. Pirot rappelle l'affaire Liancourt qui amena la condamnation d'Arnauld par la Sorbonne ${ }^{14}$. Cette annotation ne pourra s'expliquer que plus loin car elle demande que le sens d'une lecture soit précisé pour mesurer le caractère stratégique de cette annotation.

\section{Les soulignements}

Les soulignements constituent à la fois une marque horizontale et interne : la plume quitte les marges pour se confronter au cœur du discours. Le texte peut ainsi faire l'objet d'un soulignement simple ou redoublé ${ }^{15}$ mais le plus souvent cette marque de lecture est associée à d'autres marques.

\section{Les traits latéraux combinés à un soulignement}

19 Le premier niveau associe un soulignement et des traits marginaux ${ }^{16}$. Un crescendo s'observe dans la réaction scandalisée lorsque le trait latéral est redoublé et associé à un soulignement ${ }^{17}$.

\section{Le texte barré}

20 On suppose, lorsque le texte est barré, que le scandale du lecteur atteint son comble, d'autant que le texte barré se trouve le plus souvent combiné à une annotation manuscrite. Il s'agit ici pour le lecteur de Port-Royal d'évacuer, de frapper de pure et simple nullité, de «supprimer " pour ainsi dire le texte produit par l'adversaire. Il est remarquable que soit barré un des textes les plus connus de l'Apologie pour les casuistes, il sera abondamment cité par la suite, entre autres par Sainte-Beuve. Dans celui-ci, le jésuite en appelle à la protection de la Vierge car il redoute de grands fléaux pour Paris qui se ressent de ce qu'il appelle la maladie janséniste, on suppose une sorte de peste. Pour conjurer ce malheur, le jésuite offre alors à la Vierge -comme marque d'expiation - la pure et simple ruine physique de l'abbaye janséniste. Le lecteur de Port-Royal pour sa part barre, détruit symboliquement, un texte qui lui-même propose une démolition :

[...] c'est demander pardon à la Vierge du déshonneur qu'elle a reçu de ces tettres, tui promettant de dissiper le Port Royal let d'exterminer le jansénisme, et pour cet impie secrétaire, il devrait craindre ce qu'autrefois on pratiquait à Lyon envers ceux qui avaient composé de méchantes pièces : on les conduisait sur le pont et on les précipitait dans le Rhône ${ }^{18}$. 


\section{Deux hapax}

21 On s'arrêtera sur deux cas remarquables d'annotations non verbales.

22 Un développement de Pirot sur l'assassinat et marqué d'un trait latéral associé à une croix $^{19}$. Il est impossible d'affirmer que cette annotation émane spécifiquement de la plume de Pascal. On ne peut s'empêcher pourtant de rapprocher cette marque des nombreux signes cruciformes qui émaillent le manuscrit original des Pensées. Dans l'ordre symbolique et géométrique on note encore le cercle (autre marque spécifiquement pascalienne qu'on retrouve encore dans le recueil original des Pensée $^{20}$ ). Il isole un passage spécifiquement centré sur la vomissure, thème qui a couru tout au long de la campagne de Provinciales, associé le plus souvent à la question de la gloutonnerie.

\section{Les deux niveaux secondaires}

23 Les niveaux secondaires de l'annotation permettent d'aller plus avant dans la compréhension de la méthode de l'officine polémique de Port-Royal. On y décèle plusieurs lecteurs pour un même texte, chacun ayant une tâche précise que deux niveaux d'annotations secondaires permettent de définir.

\section{Les corrections internes du texte}

24 On repère un premier niveau d'annotation secondaire qui intervient de façon très précise, souvent dans les interlignes du texte : des mots sont barrés et réécrits. Il s'agit en fait le plus souvent du report intra-textuel des corrections indiquées dans l'erratum joint à l'Apologie. Ainsi l'expression « anges de Port-Royal » se trouve-t-elle corrigée en "aigles de Port-Royal ${ }^{21} »$. Ces corrections sont calligraphiées et parfois corrigent d'elles-mêmes une erreur de pagination ${ }^{22}$. On lit ici les corrections des "petites-mains » de Port-Royal qui facilitent et accélèrent la lecture des « concepteurs » de la polémique, ceux qui ont pour charge de rédiger les réponses aux textes jésuites. Il s'agit, en débarrassant le texte de ses erreurs déjà corrigées par l'adversaire, d'éviter aux lecteurs soucieux d'efficacité la perte de temps du report continuel à l'erratum mais aussi le faux-pas qui pourrait, dans l'ardeur de la volonté de répondre, les conduire à voir une faute là où il n'y en a pas.

\section{La préparation des citations}

La question de la citation est essentielle en polémique où le texte adverse, véritable «texte-patient ${ }^{23}$ ", est sans cesse cité dans une intention précise et destructrice. Le second niveau d'annotation interne témoigne d'une lecture tout aussi technique que le premier lorsqu'il porte la trace de la "préparation» du texte de Pirot en vue de citations. Une dizaine de pages $^{24}$ se trouve ainsi traitée en vue d'une citation, soit environ $5,2 \%$ de l'ouvrage. On trouve un cas typique de cette préparation des citations aux pages 56-57 du texte : la portion du texte cité se trouve placée entre deux traits horizontaux tracés dans les interlignes et dont chacun comporte un retour vertical qui permet de délimiter exactement la partie du texte à citer. La partie centrale du texte qui sera « oubliée » dans la citation se trouve barrée d'un simple trait vertical. Plus bas une seconde partie est reprise et marquée selon le même procédé. Des indications marginales indiquent que les deux parties sélectionnées par l'annotation devaient se 
trouver dans la même citation : deux B en capitales et placés entre crochets sont tracés face à chacune d'elles. Le mot «fin » indique, en marge encore, l'endroit où la citation se termine.

On sait que les citations de l'Apologie furent importantes dans les Écrits des curés. On peut dire que les citations ont eu deux fonctions : certaines furent utilisées dans le cas de reparties directes dont on verra un exemple plus bas, d'autres - comme c'est le cas ici - ont dû être sélectionnées en vue de la constitution de listes qu'on trouve dans les innombrables projets de censures de l'ouvrage de Pirot.

\section{Cas d'annotations complexes}

Dans d'autres cas la préparation du texte en vue d'une citation peut se révéler plus complexe au sens où elle peut entrer en conflit avec l'annotation marginale du texte. On peut ainsi remarquer une page où une partie du texte est barrée alors qu'elle comporte un double-trait marginal ${ }^{25}$. Il ne s'agit pas ici d'une concurrence entre lecteurs où l'un sélectionnerait le texte que l'autre barre. On y voit plus vraisemblablement la marque de la préparation de centons, pratique fréquente dans les Provinciales et les polémiques connexes. En ne sélectionnant que certaines parties du texte, celui qui cite parvient - au prix d'une sorte de montage qui n'en fait apparaître que la partie saillante - à rendre plus choquant le propos qui se disqualifie ainsi presque de lui-même aux yeux du lecteur. On peut considérer que cette technique, qui relève de la catégorie que Gérard Genette appelle le "bricolage », trahit la pensée de l'adversaire au sens où elle rend aveuglante et directe une réflexion que celui qui la formule désire nuancer en la développant de façon indirecte ${ }^{26}$. Il faut citer ici au long l'exemple de la p. 49 de l'Apologie pour les casuistes:

Le texte de Pirot est le suivant :

Supposons par exemple qu'une sœur soit dans une occasion involontaire de commettre le péché de Thamar avec son frère Annon, qu'une fille soit poursuivie par son propre père, qu'une belle sœur succombe aux importunités d'un beau-frère. Si vous renvoyez ces personnes à qui le mal déplaît et qui n'ont pas le moyen d'un sortir, vous leur mettez le désespoir en l'âme et leur ôtez le courage d'avoir recours à Dieu. D'où il arrive que le diable redoublant ses tentations achève, par les maximes des jansénistes, de perdre ceux que les casuistes eussent délivrés du mal. La doctrine des théo ${ }^{27}$ logiens a encore plus de lieu, à l'égard de ceux qui ont contracté une forte habitude du vice par des chutes réitérées de jurer, de s'enivrer et de commettre beaucoup de péchés en matière d'impureté. Car encore que l'habitude qu'ils ont volontairement contractée par les rechutes au péché leur serve d'occasion prochaine qui les porte à jurer, à s'enivrer et à d'autres mauvaises actions; souvent toutefois on ne peut pas dire que cette habitude soit volontaire, puisqu'ils la détestent et voudraient pouvoir s'en défaire. Que si en ces circonstances le confesseur leur refuse l'absolution, selon la règle des jansénistes, il faudra plusieurs fois qu'ils attendent jusques à la fin de la vie à la donner. Même quelquefois en ce temps-là, il trouvera les pécheurs en pire état que quand ils se sont présentés à lui la première fois. Au lieu que s'il leur eût donné l'absolution suivant l'avis des casuistes, la grâce des sacrements eût fortifié la faiblesse des pécheurs et les eût retirés du mal. ${ }^{28}$ Les théologiens enseignent pareillement que l'on n'est pas obligé de renoncer à une profession où l'on est en danger d'offenser souvent Dieu et même où l'on court risque de se perdre si on ne peut pas facilement s'en défaire. La pratique de l'Église sert de preuve à ma proposition. Car non seulement l'Église souffre mais elle approuve des ordres militaires qui ont fait vœu de pauvreté, chasteté et obéissance, encore que 
les occasions fassent succomber plusieurs de ces religieux. La même Église oblige au célibat ceux qui s'engagent aux ordres sacrés quoiqu'elle n'ignore pas que ces ordres servent à plusieurs occasions d'offenser Dieu.

ligature entre les deux morceaux « recollés » reste invisible dans la mesure où le mot «théologiens " permet un enchaînement entre les deux membres de la citation, à la fois sur le mot et sur la chose. L'adverbe «pareillement » qu'on trouve au début du second membre achève de rendre logique le centon obtenu en l'associant au premier dans une forme de redoublement logique. Ensuite la partie ôtée de la citation permet d'en renforcer l'agressivité. En effet, Pirot nuance son point de vue dans le tronçon supprimé. Il le débute par une concession avec « encore que " qui ôte tout caractère ingénu à sa théorie. Il indique nettement qu'il mesure la mauvaise foi du pécheur qui «rechute» dans le péché en excusant un péché d'habitude "volontaire» par une « occasion prochaine » déterminante qui l'amène presque malgré lui à commettre le mal. De plus, la partie tronquée permet de faire disparaître une accusation directe envers les jansénistes dont Pirot affirme qu'ils pratiquent un délai d'absolution étendu parfois à la vie entière du pécheur, privant celui-ci du secours du sacrement. La citation obtenue ainsi ne trahit donc pas absolument le point de vue défendu par Pirot sur la question de l'absolution dans le cas des péchés d'habitude mais elle permet à PortRoyal de produire au lecteur un texte où le point de vue contestable de l'original apparaitra dans toute sa crudité, une fois débarrassé du passage à la fois concessif, explicatif et accusateur qui l'accompagnait.

31 Jusqu'ici seules les annotations non verbales ont été décrites. Le texte de Pirot n'est pourtant pas seulement marqué par des traits et des soulignements signifiants. Des mots et des phrases marginales offrent l'ébauche d'un véritable commentaire du texte du jésuite par les polémistes de Port-Royal.

\section{Les annotations verbales}

Les annotations verbales renseignent de façon souvent éclairante sur la façon dont l'abbaye augustinienne lit les textes de ses adversaires. Il s'agit de notes de travail qui 
balisent dans les marges du texte de Pirot le parcours de ses lecteurs. Elles réservent aussi quelques surprises qui dépassent le simple cadre de l'étude des polémistes au travail.

Vingt-quatre pages de l'ouvrage de Pirot sont annotées, c'est-à-dire $12,5 \%$ de l'ouvrage ${ }^{29}$. Sans utiliser systématiquement l'abréviation elles restent le plus souvent d'une extrême brièveté. Ces notes semblent avoir plusieurs fonctions qu'il faut détailler maintenant.

\section{Baliser une lecture} dans lesquels on n'ordonne presque plus de prêtres tant les conditions y sont sévères. En marge du passage on trouve un mot dont la lecture est problématique. Ce peut-être «Beauvais » mais une comparaison avec le mot «Beauvais » qu'on trouve écrit dans la marge citée plus haut invite à une autre proposition. L'initiale diffère du point de vue graphique et semble être ici plutôt un «L» qu'un « $B$ ». La séquence centrale semble plus proche d'un «nn » que du « uu » ou « uv » de Beauvais. La fin du mot paraît plutôt un « $y$ » et diffère de la combinaison « is » qu'on trouvait à la fin du Beauvais qu'on a vu précédemment. On pourrait alors lire, plutôt que «Beauvais ", «Loannais » ${ }^{32}$, variante de Laonnais. L'annotateur supposerait ici que c'est l'évêché de Laon que vise Pirot sans le dire dans son texte. En effet César d'Estrée - un proche de Port-Royal - y fut nommé évêque en 1653. Signalons pour finir que cette note éclaircit une allusion, contrairement au «Beauvais " qui, écrit plus haut, se contentait de signaler en marge un passage explicite du texte. Les notes de balise ont une dernière fonction lorsqu'elles affichent une dimension planificatrice.

- Établir un plan de travail 
41 En marge d'un long excursus que propose Pirot sur la question de l'usure et où l'apologiste des casuistes se réfère longuement au Triple examen de Du Bail ${ }^{33}$ on lit: " tout le traité des usures est à lire ${ }^{34}$ ». Il s'agit ni plus ni moins que d'un plan de travail proposé au polémiste qui voudrait répondre à Pirot et qui indique bien quelle exigence est celle de Port-Royal dans sa stratégie de repartie. Cette note indique encore le cas qu'on fait de l'ouvrage de Pirot qui semble ici faire l'objet d'un plan de repartie savante. Une dimension supplémentaire des notes marginales consiste en des marques de pure polémique.

\section{Les notes de combat}

est différent [on peut lire aussi « difficile»] dans saint Augustin $^{38}$ ». La XVII ${ }^{\mathrm{e}}$ objection porte sur la question des valets. Selon les casuistes ils peuvent se rétribuer en volant si leur maître ne les paie pas. Pirot convoque à nouveau un exemple de saint Augustin qui « donne à entendre » qu'un médecin dont la peine n'est pas récompensée par le malade ou un artisan qui n'est point payé pour sa besogne peuvent se rétribuer et «prendre contre le gré de ceux qui doivent ». Le passage annoté sera réfuté en ce sens dans la VIII ${ }^{\mathrm{e}}$ remarque du Troisième Écrit des curés de Paris. On note ici les marques d'une collaboration de Nicole aux annotations car c'est à ce dernier qu'on attribue le plus souvent le Troisième Écrit. Ceci donnerait un poids supplémentaire à l'annotation manuscrite relevée par Faugère et identifiée comme écrite de la main de Nicole, témoin direct de la lecture de l'Apologie. Un degré peut encore être franchi lorsque le corps même de l'annotation contient un jugement de valeur. plusieurs reprises les marges indiquent une "Fausseté ${ }^{39}$ » et, franchissant encore un degré, elles peuvent affirmer que le lecteur estime que "cela est faux ${ }^{40}$ » ou qu'il se trouve face à une " étrange maxime ${ }^{41}$ ». Ces notes peuvent encore renseigner sur l'état d'esprit de l'annotateur lorsqu'il inscrit face à un passage les mots « que veut-il dire? ${ }^{42}$ ». Plus que de véritables embryons de réponses circonstanciées il s'agit surtout de réactions qui renseignent sur le « scandale » qu'a dû causer à ses adversaires la lecture $\mathrm{du}$ défenseur des nouveaux casuistes. Un dernier niveau d'annotation reste à considérer dans l'exercice de la polémique dont l'essence est d'impliquer de répondre à l'adversaire ${ }^{43}$. 

catégories. Doit-on aller jusqu'à différencier, par exemple, les plumes et les lecteurs et cela même dans le cadre des annotations non verbales. Jusqu'où aller? Jusqu'où, surtout, ne pas pousser l'investigation?

\section{Doit-on aller jusqu'à différencier les types de traits ?} personne qui les trace. Il semble bien que plusieurs annotateurs soient au travail. Peuton alors décider de qui trace tel trait ? Un type de trait, manifestement plus " tremblé " que les autres peut amener à différencier certains « traceurs ». Mais doit-on pousser la scénarisation de la lecture jusqu'à affirmer qu'une main plus "faible" parce que tremblante est au travail et que cette main fatiguée est celle de Pascal ? Au rebours, que penser des traits fermes? Sont-ils ceux d'un aide qui supplée, au cours de la lecture, à la main épuisée de celui qui n'a plus la force d'écrire? Doit-on conclure que la maladie de Pascal lui interdit d'annoter mais pas de polémiquer? On comprend ce que ces prises de position peuvent avoir de subjectif et dans quelle mesure elles sont tributaire du «scénario" de l'activité polémique de Pascal établi par la tradition critique qui traça à plaisir le portrait du Pascal souffrant. La même perplexité peut naître dès lors qu'on délaisse les annotations non verbales pour les annotations verbales. 


\section{Peut-on statuer sur les annotations verbales : qui écrit?}

53 écriture principale ${ }^{47}$. On pense que celle-ci est susceptible d'évolution. Prenons le cas de l'annotation des pages 132-133. L'annotation de la page 132 n'est peut-être pas de cette main principale mais le mot «fin» de la page 133 semble d'elle. En tout cas ces pages portent la marque - même s'il s'agit de la même main - de deux moments différents du travail de l'annotateur : il ne s'agit ni de la même plume ni de la même encre.

alors pousser plus avant la scénarisation d'une lecture à laquelle on n'assistait pas, jusqu'à donner un sens à ce qui ne peut être tranché ? La question de la graphie du même mot, le mot "fin" peut amener à une tentative de réponse. Ce mot indique marginalement la fin d'une endroit sélectionné en vue de sa citation ${ }^{48}$. Or, sur certaines de ces pages préparées on remarque la présence de plusieurs écritures concomitantes ${ }^{49}$. Doit-on penser à une équipe de lecture qui la plume à la main annote collectivement ? Doit-on postuler la présence d'un annotateur principal qui décide, in fine, de valider la citation de tel ou tel passage? Doit-on décider que cette main est celle de Pascal ? La confrontation de l'écriture principale qui annote l'Apologie avec celles du recueil original des Pensées peut laisser perplexe. L'écriture offre certes des ressemblances avec celle de Pascal mais sa relative lisibilité laisse planer bien plus de doutes que de certitudes $^{50}$. Il reste que le texte de Pirot a été annoté par des polémistes de Port-Royal, dont certains de la famille même de Pascal et que certaines annotations - lesquelles ? sont bien de l'auteur des Provinciales si l'on s'en tient au témoignage de Nicole.

On peut affirmer que l'officine de Port-Royal est une ruche où beaucoup de monde lit, peut-être en même temps, peut-être à intervalle plus ou moins éloigné, les textes adverses comme celui de Pirot. Cela conforte la vision de la préparation collective des Provinciales et des libelles afférant à la campagne des Petites Lettres. Mais on pourrait tout aussi bien postuler l'existence de plusieurs époques de lectures: l'histoire de la condamnation de l'Apologie pour les casuistes s'étend sur plusieurs années ${ }^{51}$ et on sait qu'à moyen terme l'ouvrage de Pirot fut réutilisé pour obtenir la grande condamnation des maximes émanant des casuistes dits laxistes (1679).

L'Apologie de Pirot a donc un statut unique et particulier dans l'histoire de la polémique qui est née des Provinciales. On peut dire que l'annotation que comporte l'exemplaire de la Bibliothèque Mazarine est révélatrice de la méthode polémique de Port-Royal. Mais on peut encore interroger la profondeur de cet investissement en dépassant leur seule matérialité et interroger les annotations dans une optique différente, celle du sens qu'on peut donner à cette lecture de l'Apologie pour les casuistes.

\section{Les endroits annotés : les sens d'une lecture}

L'Apologie pour les casuistes constitue un cas particulier dans les réponses aux Provinciales. Elle n'est pas absolument comparable aux Impostures ou aux différentes Réponses que les jésuites tentèrent, le plus souvent au coup-par-coup, d'opposer à PortRoyal. L'Apologie de Pirot, son nom l'indique, est une " défense » des casuistes autant qu'une accusation dirigée contre ceux qui les attaquent. Pour la première fois dans la polémique qui naquit des Petites Lettres une stratégie de défense ouverte de la casuistique moderne vit le jour, où l'on vit qu'un porte-parole des jésuites assumait certaines des propositions dénoncées comme scandaleuses dans les Provinciales morales. 
Jusque-là les réponses affirmaient à l'envi que les textes des casuistes étaient tronçonnés, mutilés et qu'ils n'avaient pas le sens permissif que leur donnait Pascal. L'Apologie, en assumant et en défendant certaines des propositions incriminées, constituait le texte idéal pour Port-Royal et les besoins de sa polémique. Le soin apporté à l'annotation va le révéler. Le sens premier qu'on peut donner à l'annotation va dans le sens de la correction que les lecteurs sont dans l'obligation d'apporter à un texte qu'ils comprennent comme fautif jusqu'à la diffamation.

\section{a. Corriger les fautes de Pirot}

\section{Les passages les plus choquants contre Port-Royal}

58 Un premier niveau d'annotation, si l'on suit le crescendo des marques d'une réaction scandalisée face aux allégations du texte adverse, marque les passages choquants en eux-mêmes, sans s'astreindre au commentaire verbal. Ainsi un passage de la $\mathrm{XVI}^{\mathrm{e}}$ objection de Pirot est-il stigmatisé d'un soulignement combiné à un double-trait latéral ${ }^{52}$. Pirot revient sur le passage de la sixième Provinciale qui affirme qu'un religieux chassé de son monastère n'est pas obligé de se corriger pour y retourner et qu'il n'est plus lié par son vœu d'obéissance. Pirot répond par un aphorisme assassin que le lecteur de Port-Royal souligne: "quand les éthiopiens deviendront blancs les jansénistes nous traiteront avec candeur ». La suite du texte porte sur le caractère antirégulier des jansénistes: dans certaines villes où ils sont puissants, affirme Pirot, on empêche la jeunesse d'entrer chez les bénédictins et les mendiants. Le lecteur se contente de souligner ce qui consiste en un ressassement maintes fois entendu au cours de la polémique anti-janséniste.

59 Parfois, d'un mot, le lecteur exprimera son mépris. Ainsi, combiné à un trait latéral et face aux «humiliations affectées » de Port-Royal «comme faire des sabots et d'autres bas ouvrages d'artisans $s^{53}$ ", on peut lire en marge: «sotises» (avec un $t$ ?) ou « songes ${ }^{54}$ ». Cette « sotise» (ou ce « songe ») peut devenir un « Étrange songe » ou une " étrange sotise ${ }^{55}$ " face à un paragraphe qui dresse, dans une virtuose attaque ad personam un parallèle entre Antoine Arnauld et Yves de Paris. Le lecteur croise alors les noms de tous les chefs de file du mouvement port-royaliste. Arnauld, sous le poids des antithèses et des oppositions, devient une sorte de créature inquiétante, véritable double faussé, inversé et satanique, de saint Yves. Un autre niveau d'annotation marque les corrections qui apparaitront dans de futurs libelles.

\section{Les corrections savantes futures}

La réponse que fait Pirot à la XXII ${ }^{e}$ objection comporte un trait latéral accompagné de cette annotation en marge : "cela est faux ${ }^{56}$ ». Il s'agit de la reprise de l'argument des juges que les casuistes autorisent à recevoir des présents. Ce cas marque bien la façon dont Pirot innove dans sa réponse: les réponses produites jusqu'ici par les jésuites lisaient une diffamation des casuistes dans ce type d'affirmation. Pirot opte pour une stratégie de renchérissement savant et affirme que c'est ici le sentiment d'Augustin. Il produit l'exemple l'Épître 54, Ad Macedonium, dans laquelle, selon Augustin, la coutume excuse ces pratiques. Ce passage précis prépare vraisemblablement la réfutation de la lecture que fait Pirot d'Augustin dans la IX remarque du Troisième Écrit des curés de Paris 
(7 mai 1658). Mais il ne s'agit pas seulement de corriger l'adversaire. La logique polémique suppose l'attaque.

\section{b. Attaquer l'adversaire}

61 Une série d'annotations vise à faire apparaitre que Pirot assume le point de vue parfois extrême que prête Pascal aux nouveaux casuistes dans les Provinciales.

\section{Montrer son extrême relâchement}

Sur la question du probabilisme, la logique défendue par Pirot est la même que celle attaquée par Pascal dans sa Provinciale. Jusqu'ici, lorsqu'ils défendent le probabilisme, les jésuites répondent aux Petites Lettres sur le mode «mineur ». Ils affirment le plus souvent que, face aux apories du tutiorisme historique qui commande de suivre l'opinion la plus probable - ce qui n'est pas toujours possible - le probabilisme consiste en une voie moyenne, plus pragmatique. Cette posture vise à faire admettre au public l'innocuité de cette «nouvelle » doctrine dont le seul but en théologie morale serait de répondre à ce qu'on appellerait aujourd'hui un vide juridique.

Pirot va plus loin et attaque frontalement le tutiorisme. Il énonce un point de vue général qui affirme qu'on n'a pas systématiquement à suivre le point de vue le plus sûr, même si l'autre solution envisagée dans le doute en morale semble acceptable. La règle que suivent les nouveaux casuistes est assumée par Pirot qui confond résolument le sûr et le probable :

dès là qu'une opinion est probable, elle est si assurée qu'on ne court point risque de se damner en la suivant ${ }^{57}$.

64 Le point de vue de Pirot confirme le pur probabilisme :

Je dis plus, à savoir que la sûreté ne reçoit point de plus et de moins, mais est indivisible lorsqu'il ne s'agit simplement que de l'action morale qui se fait dans une opinion probable. Ce qui me fait ajouter qu'une opinion probable n'est pas moins assurée qu'une qui est plus probable et que cette distinction d'opinion moins ou plus assurée ne doit avoir lieu que quand, outre l'action, on prétend la production de quelque autre chose ${ }^{58}$.

Ce passage est fortement marqué par un trait marginal. On sait l'utilisation qui sera faite par la frange dite «rigoriste» de ce type d'argument nommément condamné en 1679.

D'autres passages du texte sont remarqués. Sur l'homicide tout d'abord, un trait latéral et un soulignement partiel marquent un passage où Pirot propose de se servir de la «raison naturelle» "pour juger si une personne particulière peut tuer celui qui l'attaque non seulement en sa vie mais encore en son honneur et en ses biens ${ }^{59}$ ». Le défenseur des casuistes suggère ici que le décalogue et le cinquième commandement en particulier doivent être relus à la lumière de la loi naturelle : l'Écriture contient des récits d'homicides non criminels.

67 Sur le duel ensuite où un passage précis du texte est marqué :

Vous ne trouverez point de casuistes qui disent que, nonobstant une excommunication des papes et des évêques contre ces furieux meurtriers, il n'y a point de péché à faire ce métier de gladiateur ; quand même nous accorderions que celui refuse le combat perd un véritable honneur qu'il peut légitimement 
rechercher. La raison est que le roi et nos supérieurs sont par dessus l'honneur des particuliers ${ }^{60}$.

Paris qui se réfèrent précisément aux passages relevés par ces annotations ${ }^{63}$. Un niveau d'annotation prépare plus encore qu'une logique de stricte rétorsion dans son approche critique du texte. On repère une volonté d'attaquer les bases mêmes de la logique de Pirot.

\section{Saper la logique de Pirot}

71 On peut lire en effet l'annotation lapidaire "mauvaise conséquence ${ }^{64}$ » face à un passage où Pirot se révèle particulièrement confus. On devine que le jésuite tente, à propos de la citation d'Aristote qu'on lit à la fin de la quatrième Provinciale, de faire apparaître des divergences entre la ligne du secrétaire et celle de ses maîtres jansénistes. La "mauvaise conséquence » pointe le défaut de l'attaque de l'adversaire, fautive du point de vue logique. Mais à un autre niveau, l'annotation vise le parti polémique de Pirot qui tente d'opposer frontalement Pascal et Port-Royal : en alléguant Aristote et la lumière naturelle contre les théologiens jésuites, Pascal, selon Pirot s'opposerait aux jansénistes, ennemis de la lumière naturelle puisque théologiens de la grâce efficace. Le jésuite entend donc disloquer le front constitué par Port-Royal en opposant le rédacteur des Petites Lettres aux « jansénistes ». Un niveau supplémentaire de la divergence repose sur l'utilisation d'Aristote, philosophe, dans une querelle strictement théologique. L'argumentation peut être rapprochée - mais sous un jour réducteur et négatif - de la fameuse discussion que met en scène L'Entretien de Pascal avec $M$. de Sacy où l'on voit une opposition entre un théologien de stricte obédience et son jeune contradicteur qui considère que les philosophes peuvent être utilisés en contexte apologétique. La "mauvaise conséquence» viserait donc le fait que Pirot induit une opposition entre le secrétaire et Port-Royal qui n'existe pas ailleurs que dans son imagination. Le lecteur place ici deux mots d'une annotation qui sont comme l'embryon d'une argumentation. Il peut aller plus loin.

72 Face à un développement à forte connotation juridique on peut lire : «danger de faire passer les canonistes pour casuistes ${ }^{65} \%$. Le tour presque gnomique de l'énoncé, son caractère lapidaire, donne ici à lire un texte situé aux confins de l'annotation et de la " pensée ». Un ultime degré d'annotation reste à considérer, le moment où le scandale atteint une dimension personnelle, où les polémistes se lisent et se commentent de personne à personne. 


\section{Se confronter directement au jésuite}

73 Un niveau d'annotation indique que l'annotateur traque littéralement tous les passages qui mettent en cause l'auteur encore inconnu des Provinciales, avec qui Pirot cherche à plusieurs reprises la confrontation directe.

74 Les passages où le jésuite menace le plus directement l'auteur sont remarqués, même lorsqu'il le fait d'assez loin. Dans le scénario du scandale qu'essaie de dessiner cette étude, se devine la réaction de l'annotateur face à certains passages. Ainsi, dans le passage sur les dévotions à la Vierge qu'on a cité et commenté plus haut ${ }^{66}$, on peut lire que Pirot menace les libraires qui impriment ce qu'il considère comme des impiétés contre la Vierge. La partie du texte de Pirot est stigmatisée par un trait latéral sur toute la page. Une partie de ce texte est isolée par un trait horizontal comportant l'annotation « $\mathrm{EE}$ » en marge, la partie isolée prend fin page suivante avec un trait horizontal et « fin » en marge. Il s'agit donc d'un processus d'élaboration de citation. La fin du texte préparé en vue de la citation contient la menace grave envers Port-Royal citée plus haut mais, dans la partie retenue pour la citation une partie se trouve barrée : celle « lui [à la Vierge] promettant de dissiper le Port-Royal et d'exterminer le jansénisme, et pour cet impie secrétaire ${ }^{67} »$ ce qu'on faisait autrefois à Lyon aux auteurs de méchantes pièces: "on les conduisait sur le Pont et on les précipitait dans le Rhône ». Face à la partie barrée, on lit en marge «blasphèmes [illisible: pour? envers ?] celui qui en [les Provinciales] est l'auteur ». Ici un annotateur répond pour un auteur à un autre auteur.

Dans sa recherche de la confrontation directe avec Pirot, on remarque encore une réponse par la bravade, le défi. Ainsi, en parlant des dangers d'une trop grande sévérité dans les matières de théologie morale qui regardent les privautés accordées aux amants hors du mariage, Pirot affirme que le temps n'est plus à la sévérité. Il blâme alors les jansénistes qui contrefont les sévères et prévient qu'il pourrait

tirer un rideau qui découvrirait bien des choses, mais puisque vous nous menacez

de nous entretenir sur ce sujet, je me réserverai pour ce temps-là ${ }^{68}$.

En marge de ce passage on lit une note, difficilement lisible mais dont la leçon peut être «tirez le donc ».

On s'arrêtera pour terminer sur la page de titre. Sa note, sa faute et son motif semblent emblématique de la façon dont Port-Royal lit le texte de Pirot. Cette page comporte un titre développé :

APOLOGIE

POUR LES CASUITES [SIC]

CONTRE LES CALOMNIES

DES JANSÉNISTES

OÙ LE LECTEUR TROUVERA LES VÉRITÉS

de la morale chrétienne si nettement expliquées \& prouvées avec tant de solidité, qu'il

lui sera aisé de voir que les maximes des Jansénistes n'ont que l'apparence de la vérité : \& qu'effectivement elles portent à toute sortes de péchés, \& aux grands relâchements qu'elles blâment avec tant de sévérité.

Par un Théologien \& Professeur en droit Canon 

propose la lecture suivante :

Attribué à Mess. De St Sulpice

Pag. 78. Fausse [un mot illisible : « rumeur»?]

Comme je crois

81 La question de l'attribution de l'Apologie pour les casuistes est une question que se posent les auteurs de Port-Royal au moment de sa mise en circulation. Notons que l'ouvrage anonyme n'indique pas que les casuistes sont « jésuites » et encore moins que c'est « un Père de la Compagnie » qui, selon la formule consacrée, entreprend leur défense. On sait que juste avant les Provinciales, le duc de Liancourt avait eu maille à partir, du fait de ses amitiés port-royalistes, avec un chanoine de Saint-Sulpice. Doit-on ici supposer qu'une fausse rumeur a couru que l'ouvrage pouvait émaner des mêmes cercles? être lue comme une invitation à se reporter à la page 78 de l'ouvrage. En effet cette page, et elle seulement dans l'ouvrage, contient un passage isolé entre crochets et marqué de trois traits latéraux :

Monsieur Arnauld ne devrait point nous insulter dans ses Lettres injurieuses, parce qu'un de nos ${ }^{69}$ confrères avait, avec grande connaissance de cause, différé de donner l'absolution à un seigneur ${ }^{70}$.

On doit voir ici, dans le «nos confrères » l'origine de la fausse rumeur qui donne pour auteur de l'Apologie un proche de Saint-Sulpice. L'auteur se présente vraisemblablement, au sens large, comme un " confrère » des casuistes qui ont différé l'absolution du duc de Liancourt. Ici il est vraisemblable qu'en faisant taire la fausse rumeur et en démasquant l'auteur de l'Apologie comme jésuite, les polémistes de PortRoyal entendent porter le fer contre la Compagnie en dénonçant ce qu'ils lisent peutêtre comme une diversion de l'adversaire.

L'annotation de cette page est donc révélatrice du sens d'un acte de lecture en ce qu'elle corrige les fautes d'impression et renvoie au corps même de l'ouvrage qu'elle entend citer en adoptant, face à un texte qui - sous couvert de désabusement - trompe ses lecteur, une stratégie de clarification et d'accusation.

La tentative de déchiffrement des annotations de l'Apologie pour les casuistes comporte divers enseignements. Elles portent la marque du "scandale ", celui qu'éprouvent les lecteurs de la partie adverse. Elles indiquent encore - par leur précision - avec quelle attention on pouvait, à Port-Royal, lire les textes émanant de l'ennemi. Même si l'on ne 
se risquera pas à identifier l'écriture de l'annotateur principal ou à affirmer que tel ou tel trait (figure géométrique ou soulignement) émane bien de sa main, on tient que ces marques sont exemplaires de la pratique polémique du groupe qui, à Port-Royal, s'opposa aux jésuites et aux casuistes relâchés. Elle est caractérisée par le sérieux et l'attention accordée aux textes de l'autre à qui l'on s'oppose en tout mais qu'on ne prend certainement pas à la légère.

\section{NOTES}

1. Septième écrit des curés de Paris, dans Pascal, CEuvres complètes, éd. Michel Le Guern, Paris, Gallimard, «Bibliothèque de la Pléiade », 1998, t. I, p. 914. Nous désignerons par la suite cette édition sous le sigle LGP I.

2. Apologie pour les casuistes contre les calomnies des Jansénistes, Paris, 1657, 191 p. in $4^{\circ}$.

3. Le lecteur trouvera dans notre ouvrage, « Rien ne nous plait que le combat, La Campagne des Provinciales de Pascal », Clermont-Ferrand, Presses Universitaires Blaise Pascal, 2007, p. 675-750, une analyse de l'Apologie de Pirot et de la polémique qui naquit aussitôt après sa mise en circulation ainsi que des renseignements bibliographiques.

4. Apologie pour les casuistes, p. 2.

5. Voir Godefroy Hermant, Mémoires [...] sur l'histoire ecclésiastique du xVII siècle (1630-1663), éd. Augustin Gazier, 6 vol., Paris, Plon, 1905-1910, vol. IV, p. 106.

6. D'après Barbier, Sommervogel, VI, col. 856-857, les annotations marginales de l'exemplaire de la Mazarine sont de Blaise Pascal.

7. Sur le passage de la p. 171-172 de l'Apologie.

8. Sur l'histoire du fonds Faugère qu'on suit de la famille Périer à Saint Jean d'Angély puis de Prosper Faugère à la Bibliothèque Mazarine, voir l'introduction générale de Jean Mesnard aux Euvres complètes de Pascal, Paris, Desclée de Brouwer, 1964, t. I, p. 113-133. Le tirage de l'Apologie sur les casuistes est décrit p.129, Jean Mesnard y reconnaît l'écriture de Nicole et celle des membres de la famille Périer dont étienne, Florin et Louis.

9. L'exemple des annotations des pages 18 et 23 de l'Apologie est significatif. Il peut s'agir de la même écriture : voir les «f » (p. 18), comparer le « de » (p. 23) avec le « de » de la p. 77. Voir encore le «impiété » de la p. 38 à comparer avec la séquence «te »du «fausseté » de la p. 13. Mais il y a des cas où on ne peut être sûr : p. 60 ou p. 132 par exemple.

10. Voir par exemple la p. 84 : doit-on intégrer la $X X X^{e}$ objection de Pirot dans le cadre de l'annotation? Le cas de la p. 113 doit être considéré : l'ordonnance de Blois entre-telle dans le cadre de la réaction du lecteur?

11. Apologie, p. 119.

12. Ibid., p. 185.

13. Ibid., p. 153. 
14. Ibid, p. 78.

15. Ibid, p. 3, p. 26.

16. Ibid, p. 6, p. 130.

17. Ibid, p. 129.

18. Ibid, p. 133.

19. Ibid, p. 67.

20. Voir Jean Mesnard, " Nombres et textes figurés chez Pascal », XVII siècle, $\mathrm{n}^{\circ} 177$, Pascal, octobre-décembre 1992, p. 521-532.

21. Apologie, p. 31.

22. Ainsi à la p. 31 de l'Apologie un "former » apparaît, corrigé dans le corps du texte alors que dans l'erratum il est signalé à la p. 32.

23. Sur cette notion voir Dominique Maingueneau, Sémantique de la polémique : discours religieux et ruptures idéologiques au XVII siècle, Lausanne-Paris, L’Âge d'homme, 1983.

24. Apologie, p. 49, 56, 57, 58, 60, 132-133, 162, 175-176.

25. Ibid., p. 49.

26. Voir, entre autres, Roger Duchêne, "Citation trahison ", dans L'Imposture littéraire dans les Provinciales de Pascal, [1984], deuxième édition augmentée, Aix-en-Provence, Publications de l'Université de Provence, 1985, p.171-184 et Antoine Compagnon, La Seconde Main ou le travail de la citation, Paris, Seuil, 1979, p. 18-19.

27. Cette partie fait l'objet d'un soulignement hachuré.

28. Cette partie est biffée par quatre grands traits obliques.

29. Apologie, Titre, et p. 8, 9, 10, 11, 13 (3 annotations), 16, 17, 18, 23, 35, 38, 60, 73, 77, 81, 97, 99, 125, 129, 132, 133, 145, 162.

30. Ibid., p. 10.

31. Ibid, p. 125.

32. Ibid, p. 73.

33. Louis Bail, De Triplici Examine ordinandorum, confessariorum et poenitentium, cum appendice de censuris et impedimentis canonicis, Paris, Pierre Chevalier, 1648.

34. Apologie, p. 99.

35. Ibid., annotation de la p. 23.

36. Ibid, p. 23.

37. Blaise Pascal, éd. citée, t. I, p. 836.

38. Apologie, p. 81. Sans revenir trop longuement sur la doctrine augustinienne et sa valeur emblématique à Port-Royal, on note que cette note pourtant indique bien que la polémique qui oppose les jésuites à leurs adversaire consiste pour une bonne part à produire deux interprétations exclusives et contradictoires de l'autorité de ce Père incontournable au cours d'une querelle d'appropriation où les uns et les autres produisent à destination de public de la polémique une lecture d'Augustin visant à invalider celle de l'autre.

39. Idem, p. 8 et passim.

40. Ibid, p. 97.

41. Ibid, p. 129. 
42. Ibid, p. 77.

43. Voir Dominique Descotes «Le rapport des parties dans les Provinciales », dans Pascal, Corneille, désert, retraite, engagement, Actes de Tucson, édités par Jean-Jacques Demorest et Lise Leibacher-Ouvrard, Biblio 17-21, PFSL, Paris, Seattle, Tübingen, 1984, p. 11-36.

44. Apologie, p. 13.

45. Laurent Thirouin, «Imprudence et impudence. Le dispositif ironique dans les Provinciales », Courrier du CIBP, 18, 1996, p. 31-42.

46. Apologie, p. 46 et p. 63 ou 69 par exemple.

47. Voir un échantillon à la p. 60.

48. Apologie, p. 49, 57, 60, 133, 162.

49. Ibid., p. 162.

50. C'est l'avis de Laurent Susini et de Gilles Proust que je suis heureux de citer et de remercier ici pour leurs réponses. Gilles Proust écrit: «Si je dois vous donner une réponse aujourd'hui, je commencerais par vous dire qu'il est matériellement impossible d'affirmer que certaines de ces annotations sont de Pascal. En effet, comme vous pouvez le constater vous-même dans l'édition phototypique de Brunschvicg, l'écriture de Pascal est très variable selon qu'il écrit pour lui-même (les Pensées) ou quelqu'un d'autre (des lettres). Les notes et les textes de Pascal dans le corpus des Pensées sont écrits de façon quasi illisible (d'où la nécessité des Copies). Quelques mots parfois sont un peu plus lisibles en particulier lorsqu'il écrit plus gros (c'est notamment le cas des titres) mais cela reste limité à quelques mots. [...] C'est d'ailleurs la petitesse de votre corpus et son hétérogénéité qui empêchent tout diagnostic. Tout ce que je puis faire est de vous donner une impression subjective : le mot « fausseté » est peut-être de Pascal, rien ne s'y oppose ; en revanche, le texte "ce passage est difficile dans st Augustin » n'est pas de Pascal : l'écriture de Pascal est plus arrondie et plus irrégulière ; le texte "Attribue a Mss de st Sulpice... » est plus proche de l'écriture de Pascal mais il me semble que celle de Pascal est plus penchée si je me base sur le document présent dans C1. Globalement, mon impression serait plutôt négative si on considère qu'une grande partie des commentaires en marge sont écrits par une même personne. Ma conclusion est que je ne peux rien en conclure. Prudence? trop peu d'éléments d'un côté, trop d'éléments variables de l'autre». Laurent Susini émet lui aussi de forts doutes: "l'attribution à Pascal ne me paraît pas du tout aller de soi, l'écriture ne me semble ni assez rapide, ni assez simplifiée, et les jambages pas assez agressifs. En même temps, il $\mathrm{y}$ a les doubles « $\mathrm{s}$ » qui me disent quelque chose, et les « $\mathrm{f}$ » aussi ».

51. Le texte ne sera condamné qu'en 1659, voir Jean Mesnard, «La collaboration des écrivains de Port-Royal aux censures contre l'Apologie pour les casuistes, (1658-1659) », Chroniques de Port-Royal, 1983, p. 3-20.

52. Apologie, p. 80.

53. Ibid., p. 11, 1.7 à 13.

54. Ibid, passim.

55. Ibid, p. 16.

56. Ibid, p. 97.

57. Ibid, p. 46. Plus loin le défenseur des casuistes affirme que « quand il n'est question que de l'action morale, toute opinion probable est aussi sûre que les autres, qui ont plus de probabilité », p. 47.

Courrier du Centre International Blaise-Pascal, 38-39 | 2017-2018 
58. Idem.

59. Ibid, p. 88.

60. Ibid, p. 94.

61. Ibid, p. 129, avec double soulignement manuscrit.

62. Idem.

63. Factum pour les curés de Paris, Pascal oeuvres complètes, LGP, I, p. 839-840.

64. Apologie, p. 9.

65. Ibid., p. 18.

66. Ibid, p. 131-132.

67. Ibid.

68. Ibid, p. 144.

69. Ici le «vos confrères" imprimé est corrigé de façon manuscrite en «nos confrères ", conformément à la leçon de l'erratum.

70. Ibid, p. 78.

INDEX

Mots-clés : Pirot (Georges), Port-Royal, Provinciales

Keywords : Pirot (Georges), Port-Royal, Provinciales

\section{AUTEUR}

\section{OLIVIER JOUSLIN}

Olivier Jouslin enseigne la littérature en première supérieure au lycée Louis Thuillier d'Amiens. Il a soutenu sa thèse sur Pascal et le dialogue polémique en 2004 à l'Université de Paris IV Sorbonne 